\title{
Corrigendum: An Integrated Management Model of Patients With Atrial Fibrillation: The Experience of the Local Health Unit Tuscany North-West
}

Casolo G, Cavirani G, Del Carlo A, Iannazzo S, Pardini M, Squillace A. An Integrated Management Model of Patients With Atrial Fibrillation: The Experience of the Local Health Unit Tuscany North-West. Farmeconomia. Health economics and therapeutic pathways 2019; 20(Suppl 1): 3-16; https://doi.org/10.7175/fe.v20i1S.1454

There was an error in the description of GLORIA-AF registry program at page 10 in this Supplement by Casolo et al. [Farmeconomia. Health economics and therapeutic pathways 2019; 20(Suppl 1): 3-16; https://doi.org/10.7175/fe.v20i1S.1454]. The online version has been corrected on February 12, 2020, as shown below.

The paragraph «GLORIA-AF is a prospective registry program describing antithrombotic treatment patterns in patients with newly diagnosed NVAF at risk of stroke. The study of Huisman et al. [21] aimed at describing data of phase 2, which began when the first NOAC (dabigatran) became available and comparing them with data of phase 1 (pre-NOAC era). Phase 1 involved 1,063 patients, of whom 32.8\% received VKA, 41.7\% acetylsalicylic acid, and $20.2 \%$ did not receive any antithrombotic therapy. In phase $2(n=15,092)$, the prescription of oral anticoagulant drugs has increased $(79.9 \%$ of patients, of whom $47.6 \%$ received NOAC and $32.3 \%$ VKA) while $7.9 \%$ of patients remain undertreated. Europe-related data (Figure 3) confirm the improvement of anticoagulation therapy (64.1\% in phase $1 \mathrm{vs} 89.8 \%$ in phase 2): particularly in phase 2, treatment with NOAC was more common than VKA $(52.3 \%$ and $37.8 \%$, respectively).» has been replaced by «GLORIA-AF is a prospective registry program describing antithrombotic treatment patterns in patients with newly diagnosed NVAF at risk of stroke. It comprises three phases: phase 1, before the introduction of NOACs; phase 2, during the time of the introduction of dabigatran, the first NOAC; and phase 3 , once NOACs have been established in clinical practice (still ongoing). Phase 1 involved 1,063 patients, of whom $32.8 \%$ received VKA, $41.7 \%$ acetylsalicylic acid, and $20.2 \%$ did not receive any antithrombotic therapy. The phase 2 aim was to describe the effectiveness and safety of dabigatran etexilate over 2 years from routine clinical practice in GLORIA-AF patients who are newly diagnosed with NVAF and at risk of stroke. In phase $2(n=15,092)$, the prescription of oral anticoagulant drugs has increased $(79.9 \%$ of patients, of whom $47.6 \%$ received NOAC and $32.3 \%$ VKA) while $7.9 \%$ of patients remain undertreated. Europe-related data (Figure 3 ) confirm the improvement of anticoagulation therapy (64.1\% in phase 1 vs $89.8 \%$ in phase 2): particularly in phase 2, treatment with NOAC was more common than VKA $(52.3 \%$ and $37.8 \%$, respectively)».

We apologize for any inconvenience caused. 